\title{
A Simulation Modeling Approach of Aspect-oriented Production System
}

\author{
Xiao-gao YU \\ HuBei University of Economics, Wuhan, China \\ tecom_sam@163.com
}

\begin{abstract}
Keywords: Production System, Simulation Modeling Approach, Object-oriented technology.
Abstract. For the deficiencies of the mature view of current object-oriented simulation modeling of production system, the UMLs of aspect-oriented extension, the Advanced Properties-based UML extension mechanism is completed. in this article from the aspect-oriented modeling point of view, give the cell design method which is based on the AO, mainly solute the identification of cross-cutting concerns, the selection of connection point and aspects separation, extended UML design and modeling and so on. This study provided a new way of thinking on the development of manufacturing simulation system.
\end{abstract}

\section{Introduction}

The object-oriented technology has been widely applied in simulation modeling of production system in many fields for the moment [2]. The ultimate goal of the manufacturing system simulation is optimizing the production system performance indicators, and accurately meets the market of the customer needs. So, required the closely integrated approach of using the object-oriented technology and Artificial Intelligence, expert systems, decision-making system, get the optimization analysis of the system based on the simulation[1]. The typical optimization goal such as: manufacturing time, costs, bottlenecks, and anomalies and so on, that embodied in the traditional OO modeling is often necessary to introduce a special type of treatment to deal with. Clearly, the complexity relationships of this type of treatment and entity class would increase the number of unfavorable factors of the analysis of systems. Aspect-oriented[4] Modeling (Aspect Oriented Modeling, AOM) would very well make up for this deficiency. Accordingly, this paper presents the application of AOM to support the development of manufacturing system object across multiple modules or cross-cutting behavior, improve the modeling method of the existing object-oriented simulation (OOS), and use the extension of UML to complete the specific work; finally it gave an example of a production line simulation system.

\section{Object-oriented modeling and issues of crosscutting concerns}

The OO Modeling of Manufacturing System Simulation. Object-oriented simulation modeling approach is adopted to describe the target system function, structure, information and control features of the system, and by describing the object of manufacturing features, the target behavior and the interrelationship between system modeling to achieve the separation of manufacturing system logistics, information flow and control strategy, to fully reflect the dynamic process and characteristics of the random manufacturing systems, which for discrete manufacturing systems are a powerful tool for modeling, strong support the analysis and design for manufacturing systems[3] .

In the establishment of manufacturing system simulation model, they should combine the methods of the traditional description of dynamic behavior of discrete event systems modeling and the model can be expanded and the reusability of object-oriented, to analysis of manufacturing system members of the organizational level, behavior and interactions, from the structure, function, information, control and so on, then established a unified object-oriented simulation model.

Through the abstract of manufacturing system, you can set up basic application object model, and can provide a unified description of the system input, resources, operations, output analysis, then the production system level manufacturing system simulation object class is divided into four main 
categories, namely, the physical object class, information / control object class, service and organization of the target object class categories, as shown in Fig. 1.

(1) The physical object class: the physical information resources of the system, including equipment (processing equipment, transportation equipment, robots, fixtures, buffers, working platforms, AGV track, etc.), processed parts, operation workers, etc.;

(2) Information / control object categories: Process, production planning, control rules, the running process control of production line, logistics control, fault monitoring and scheduling, workers, etc.;

(3) Service Class: Production line balancing, bottleneck analysis, hours of work analysis, human engineering analysis, the running the simulation results of production line, express the results in various forms of chart.

(4) Organization objects class: the factory, workshop, production line.

According to the manufacturer and operating characteristics of the system, all object links. According to the operation principle of a specific manufacturing environment control scheduling to coordinate, and form a specific functional behavior of dynamic manufacturing systems.

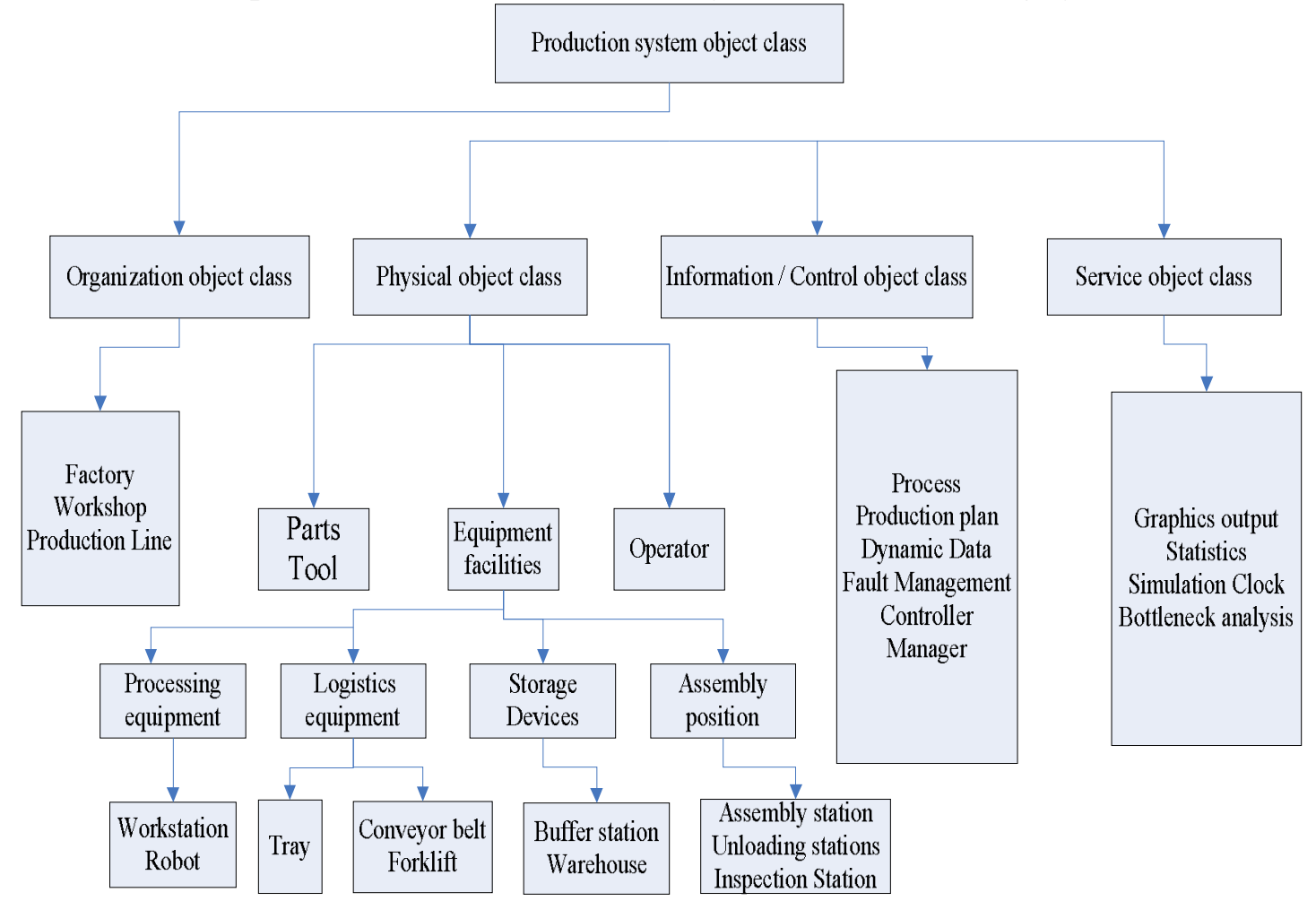

Fig.1 Manufacturing System Object Model Framework

Issues of crosscutting concerns in production system. The virtual manufacturing systems which is based on the Object-oriented method, use strict type package, many entities in the simulation have some of the same properties and methods, it allows different types that exist in a large number of the same data and behavior, compared with the vertical core questions are multiple types of cross section, and this is the cross-cutting concerns (Crosscutting concern).

When these categories are not the same super-category of generalization, then these data and methods that exist in different types of application-specific is difficult to reuse. And, as a result of class-specific application code overly complex and will enable the system to safeguard the latter becomes very difficult, making changes for a specific simulation mission will also be extremely difficult, which will affect the system robustness. The cross-section data can give the definition of a super-category, by using the type of inheritance to solve the problem, but the development and the complex of maintenance should not make it a fundamental solution to this problem.

As a result of the Organization object class don't attribute the existence of the cross-cutting concerns, manufacturing system problem concerns mainly concentrated in the physical object class, information control object class and services object class. For example, almost all of the physical object 
class attributes the existence of time and fault methods, by using the OO modeling Ways, different types of physical objects are difficult to mutually share of other types of data and methods; when the actual system performance improvements required to upgrade to the simulation system, it need to improve the different physical properties of the corresponding object class method, the work is very heavy; the existence of the certain control information object class and service object class in the system, increase the complexity of the relationship between the class.

\section{Aspect-oriented modeling based on high-level attributes of UML}

The object life cycle modeling of the state diagram of UML, described the object at different times on the dynamic behavior. If a time period to meet the conditions of the state to maintain a constant, if not satisfied then the trigger events would happen, event re-activation of the state of migration, the process of transfer of the subsidiary can perform the action, the basic form is Event [Condition] / Action. In this process the object can be seen as an isolated entity which is used to detect events and communicate with the outside world, the incident is the note for the important things are happened in the time and space.

UML is the industrialized standards of object-oriented modeling, the expansion of UML that realize aspect-oriented system modeling is a very meaningful research direction[4]. UML provides a wealth of expansion mechanism for the AOM, it can adapt to different types of methods and fields, and through the introduction of stereotypes to achieve expansion. Constructive <<aspect>> is the UML Class element model, is used to express concern of system; <<crosscut $\rangle>$ is the connecting element model of UML, is used to express he relationship of the cross section and the core functional components; $<<$ control>> is the connecting element model of UML, is used to express the controlling relationship of core categories; <<advice>> is the element model of UML, is used to express the type of element characteristics; <<joinpoint>> is the element operation model of UML, is used to express the connection point of the core category; <<pointcut>> which is the operation element model of UML is composed of a series of joinpoint.

Pointcut. For adding cross-cutting behavior by the weaving rules in the particular connection point, first of all it should capture the connection point which is required, the pointcut is used to capture and identify these connection points in the flow of procedures. Pointcut is expressed by the expansion of the structure of type <<pointcut $>$.

Pointcut has the name of the identifier and visibility, it can also have a number of contextual parameters, if not with the abstract aspects (Abstract Aspect) it should be designated to select the connection point, in the figure the modification before Definition includes public, private, protected, abstract and final. If an entry point declared as abstract, its entry points should also be declared as abstract, and the entry point can be overloaded by the pointcut with the same parameters and the same name in the "sub-area" (SubAspect); if the statement is final, it should not be overloaded, the pointcut in the sub-area should not contain the same name.

The powerful mechanism of the scope of the connection of pointcut is the use of wildcards and indicator, the expression can be used universally in the definition of <<pointcut>>, please refer the wildcards and specific indicator approach to the literature [5].

Aspect. Aspect is the basic unit of modular and localized cross-cutting concerns. Therefore, in the process of modeling, the aspect should be closed as an independent entity. In the aspect-oriented modeling, it should expand a new concept of structure type <<aspect>>. The construction inherit the structure of "Class" of standard UML, it can contain a variety of cross-cutting elements, such as pointcut, information and their own methods and data members, but also can participate in an external link and inheritance. 
The expansion purpose here is simply to complete the simulation system for modeling, so it would not describe the semantic of <<aspect >> too much. In aspect-oriented thinking, an aspect is a container of the partial cross section, it has been enough to express cross-cutting modular behavior, if it continues to conduct cross-cutting and thinning Visualized expression system, it will make the system chaos. From the general sense, the container only played the role of organizations; it can permit the simple relationship.

The relationship between the aspect and container is realized by the associated class of $<<$ pointcut $>>,<<$ pointcut $>>$ defines the connection point set which is impacted in the core container $<<$ aspect>> (Fig. 2). The pointcut is realized by the design of an abstract connection point selector association class, whose major role is to define the collection of connection point.

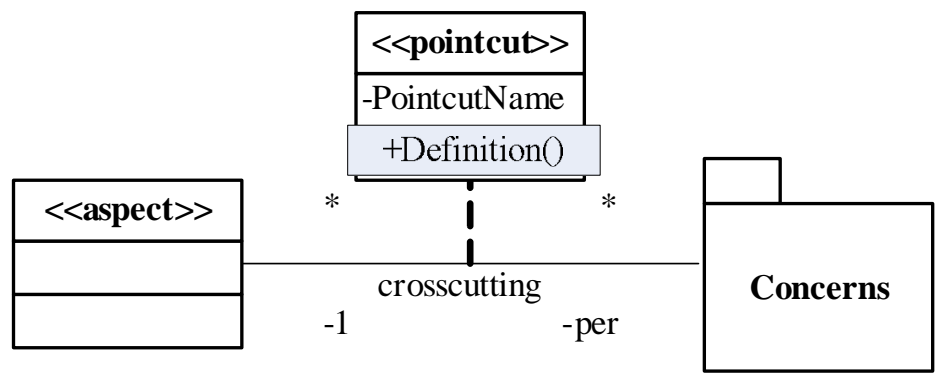

Fig.2 Aspect and Concerns

\section{An modeling example of production line simulation system}

Brief introduction of production line simulation system. A production line simulation of the process simulation system include six modules: Parts processing, quality inspection, painting, assembly parts, assembly and commissioning, finished temporary shipped. The entire simulation system users include the production director, workshop director, workshop planning and design staff, and simulation staff and so on. As a result of the division of responsibilities, different simulation system users using different simulation modules, for example, the production director can use the entire simulation module, but the machining workshop director is concerned only about the machine parts processing module.

As a part process of the simulation system an example, the processing starting point is "generator parts" object, firstly the generator parts and components transferred Job Shop Scheduling, and operational plans have to be processed in chronological order parts, if the input buffer have space, then put the parts into the buffer, the buffer object to determine the status of processing workstations, If it is being processed, wait; if it is free, enter the parts to be processed. At this time processing workstation determines the type of parts and transferee them to the machining process. After processing, send the parts to the output buffer, output buffer is the next process input buffer, and then enter the next process processing workstation, finally, put the parts into the traps, then this production line simulation is end. In accordance with $\mathrm{OO}$ modeling methods, we can set up corresponding use case diagram, class diagram, state diagram and so on, thus complete the first step described in the section of modeling.

The modeling of maintenance cross-cutting concerns. In the leaves production line processing simulation system which is described in the previous section, there are a lot of cross-cutting concerns; it mainly includes exception handling, security checks and so on, as the public nature of the demand for most categories of regular cross-section to make it easier to distinguish, summarized in the corresponding area. Now take the common exception handling module as an example. Exception handling module is an important control system components, when the exception handling operations are mapped to the processing unit, its simulation of operation will be widely distributed in the physical object class. The failure and maintenance of these two relatively simple simulation operations will be across many categories, in the simulation system there are many different types of physical objects which are packaged with the same act, so it is a cross-cutting concerns. 
Like the separation of concern time and form a time aspect, the failure concern points achieve the failure characteristics. So the system failures will not spread in various modules, by centrally managing all the system failure characteristics, it's easy to realize and maintain. In the leaf structure model of processing system, by increasing FailureAspect stereotypes to express the system failure.

\section{Aspect-oriented expansion of eM-plant based on component structure}

eM-Plant[6] (formerly known as SIMPLE + +) which is developed by Israel Tecnomatix is mainly used in production systems and production process modeling and simulation software system. eM-Plant use the object-oriented modeling (object-oriented modeling) programming method. eM-Plant software provides a rich class library interfaces and expansion mechanisms. Components are the smallest unit platform of eM-Plant, in eM-Plant much processing module are components. Each component is responsible for completing the simple or complex functions. In addition, the current aspect-oriented programming technology has achieved great development, and software components are becoming increasingly sophisticated technology. The AO expansion of the software is primarily by the expansion method of components.

The expansion components include aptamer modules, configuration files, AO sub-module. Aptamer module and the original components are the relationship of calling and being called. And passive components which are called relations, that is, it's called by the being interface; and active components are called relations, that is, it provides interface. Configuration file is an XML document, which includes the basic information of the plug-ins, including plug-in name, the only ID, version number, notes, and the relationship with others as well as plug-in interconnection pattern. Interconnection mechanisms includes that the expansion point which is provided by it and the expansion of the other plug-ins expansion point realized by it. AO aptamer module selects the cross-cutting concerns to deal with, then model it as an aspect, and realize it a subset of components. The expansion of the structure of components and the relationship of their interaction with the system components is shown in Fig. 3.

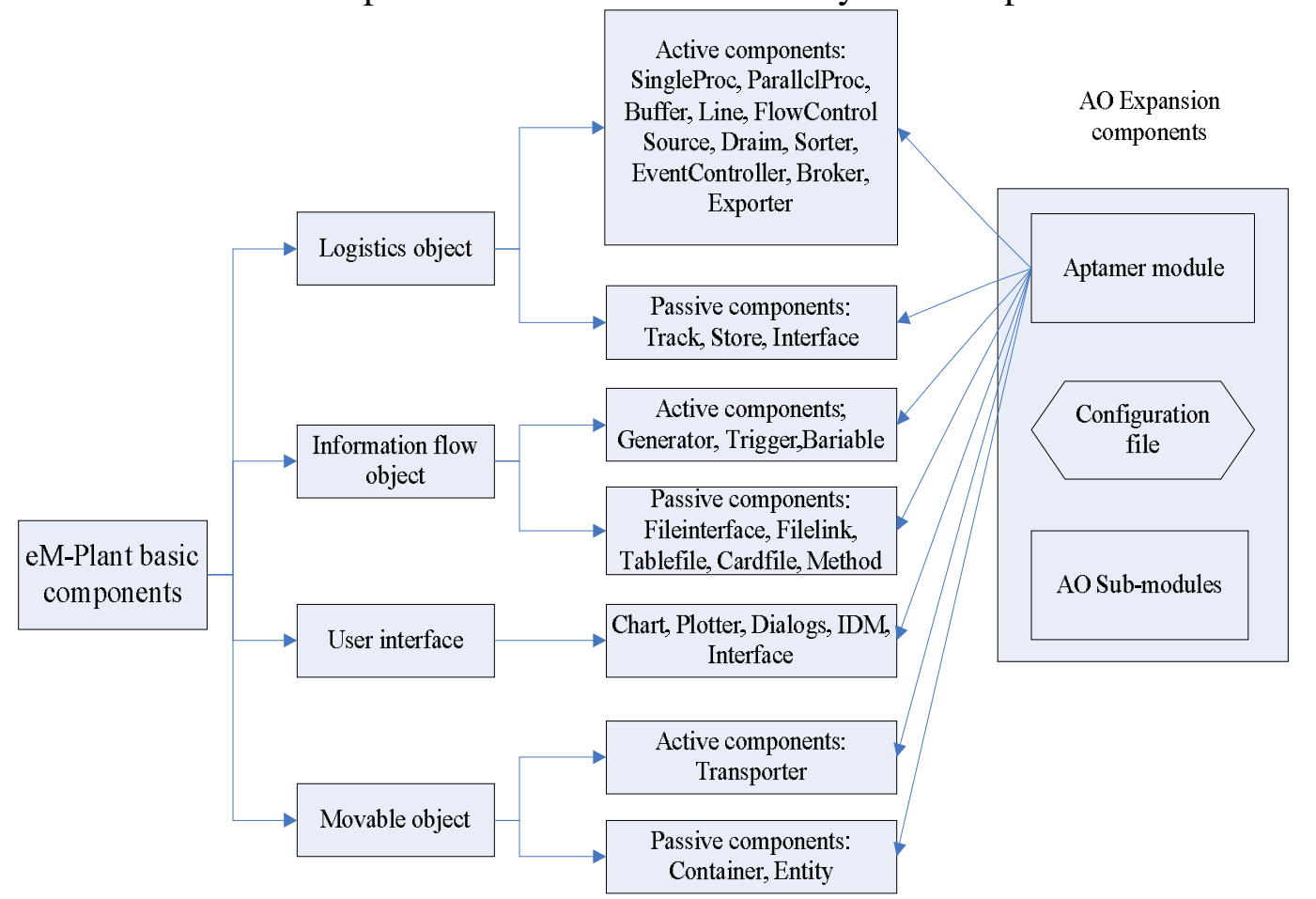

Fig. 3 the relationship of the expansion components and eM-Plant system components

\section{Conclusions}

Aiming at the deficiencies of the more mature view of current object-oriented simulation modeling of production systems, as an example of the typical maintenance cross-cutting concerns, the 
aspect-oriented modeling method of the production line simulation system is studied by using the expansion of UML. Aiming at the inadequacy of the existing simulation software, extension method of eM-Plant is proposed based on component-based structure. Because of existing restrictions on software products, in the paper the study of the production line modeling and simulation which is based on aspect-oriented thinking is still in the modeling part, and only gives the expansion of eM-Plant-oriented aspects at the design level. Next job, I will select one category to consider how to deal with the concern-points, and then will be for its modeling, by using the structure shown in the four, realize a plug-in prototype, incorporate it into the eM-Plant software.

\section{Acknowledgements}

This paper is supported by the Fund of humanities and social sciences of Hubei Province (Project No. 15D086).

\section{References}

[1] JI Ye, WEI Dog, FAN Xi-min. The Simulation of Failure Scheduling Management for Auto Product Line[J]. Computer Integrated Manufacturing Systems, 7(2011) 53.

[2] Hu Jihan, Hu Mequn, Wag Xu. Object-Oriented Design in Simulation Software Development [J]. Journal of System Simulation, 16(2014)2715-2717, 2720.

[3] HOU Yan, FAN Xi-min, YAN Ju-qi. Simulation-Based Manufacturing System Object Modeling[J]. Computer Integrated Manufacturing Systems, 7(2011) 42-46

[4] Aldwd O, Eld T, Bad A. UML profile for aspect-oriented software development[R]. Proc of the 3rd International Work-shop on Aspect-Oriented Modeling(2013).

[5] Rmavas Ldad, Aspect in Action. Manning Publications Co.(2013).

[6] Celo, G., Cot, A., Fichera, S.. Scheduling of unrelated parallel manufacturing cells with limited human resources[J]. International Journal of Production Research, v 46, n 2, January, 2014, p .405-427 